\title{
Unsteady Bioconvection Squeezing Flow in a Horizontal Channel with Chemical Reaction and Magnetic Field Effects
}

\author{
Qingkai Zhao, ${ }^{1}$ Hang Xu, ${ }^{1}$ and Longbin Tao ${ }^{2}$ \\ ${ }^{1}$ Collaborative Innovation Center for Advanced Ship and Deep-Sea Exploration (CISSE), State Key Lab of Ocean Engineering, \\ School of Naval Architecture, Ocean and Civil Engineering, Shanghai Jiao Tong University, Shanghai 200240, China \\ ${ }^{2}$ School of Marine Science and Technology, Newcastle University, Newcastle NE17RU, UK \\ Correspondence should be addressed to Hang Xu; hangxu@sjtu.edu.cn
}

Received 22 November 2016; Revised 29 December 2016; Accepted 4 January 2017; Published 24 January 2017

Academic Editor: Mohsen Sheikholeslami

Copyright (C) 2017 Qingkai Zhao et al. This is an open access article distributed under the Creative Commons Attribution License, which permits unrestricted use, distribution, and reproduction in any medium, provided the original work is properly cited.

\begin{abstract}
The time-dependent mixed bioconvection flow of an electrically conducting fluid between two infinite parallel plates in the presence of a magnetic field and a first-order chemical reaction is investigated. The fully coupled nonlinear systems describing the total mass, momentum, thermal energy, mass diffusion, and microorganisms equations are reduced to a set of ordinary differential equations via a set of new similarity transformations. The detailed analysis illustrating the influences of various physical parameters such as the magnetic, squeezing, and chemical reaction parameters and the Schmidt and Prandtl numbers on the distributions of temperature and microorganisms as well as the skin friction and the Nusselt number is presented. The conclusion is drawn that the flow field, temperature, and chemical reaction profiles are significantly influenced by magnetic parameter, heat generation/absorption parameter, and chemical parameter. Some examples of potential applications of such bioconvection could be found in pharmaceutical industry, microfluidic devices, microbial enhanced oil recovery, modeling oil, and gas-bearing sedimentary basins.
\end{abstract}

\section{Introduction}

The unsteady squeezing channel flow, caused by the moving boundary under the influence of external normal stresses or vertical velocities, is often encountered in fabrication of hydrodynamical machines, accelerators, compression and injection moulding, lubrication equipment, and polymer processing and so on. Since Stefan [1] initiated the investigation of squeezing flow by using the lubrication approximation, many researchers successively considered squeezing flow problems for various geometrical configurations in many different ways. Moore [2] concluded that the Stefan equation [1] is inadequate to describe the molecular mechanism of viscosity of squeezing flows; instead, he indicated that the influences such as surface finish, viscoelastic liquids, elastomeric surfaces, and molecular effects also need to be considered partially or entirely based on the degree of complexity of problems. P. S. Gupta and A. S. Gupta [3] noticed that the unsteady squeezing channel flow problem could be simplified significantly via similarity variables in the case that the distance between the paralleled plates varies as the square root of a linear function of time. Duwairi et al. [4] considered the heat transfer effects on the unsteady squeezing channel flow, in which case they assumed that the paralleled walls are heated uniformly by a constant temperature. The unsteady squeezing flow problems were further investigated by different researchers with consideration of various physical problems and computational techniques, such as Verma [5], Mustafa et al. [6], and Hayat et al. [7].

It is known that a large amount of microorganisms are suspended in appropriate aqueous environments, such as oceans and rivers, puddles, and droplets. Their behaviours govern the dynamics of many of these interactions with fluid mechanics. The hydrodynamic characteristics of single-celled microorganisms which are motile and self-propelled were firstly studied by Pedley et al. [8] and Pedley and Kessler [9]. Pedley's theory [8] was then adopted by Kuznetsov [10] to consider the thermal bioconvection, in which a macroscopic convective motion was induced in a fluid layer by the combined effect of density stratification caused by 
the upswimming of oxytactic microorganisms and heating from below. Kuznetsov [11] then investigated the influences of nanoparticles on the behaviours of gyrotactic microorganisms and concluded that nanoparticles can be either stabilizing or destabilizing the density of microorganisms, depending on whether the basic nanoparticle distribution is bottom-heavy or top-heavy. His idea was confirmed by $\mathrm{Xu}$ and Pop [12] via an analysis of a mixed bioconvection nanofluid flow over a stretching flat sheet with uniform free stream with an improved nanofluid model proposed by Kuznetsov and Nield [13]. The novel behaviours of thermobioconvection in nanofluids were further investigated by $\mathrm{Xu}$ and Pop [14] and Raees et al. [15].

Physically, magnetic fields can induce currents in a moving electrically conductive fluid, which in turn polarizes the fluid and reciprocally changes the magnetic field itself. Such fluid was named as magnetohydrodynamics (MHD) by Alfvén [16]. Since MHD flows have been found to be very useful in heat and mass transfer progresses in many industrial procedures such as polymer extrusion, drawing of copper wires, continuous stretching of plastic films and artificial fibers, hot rolling, wire drawing, glass-fiber, metal extrusion, and metal spinning, many studies [17-27] have been done towards understandings of their transport mechanisms and novel applications. It is worth mentioning that Khan and Makinde [28] conducted an investigation about the magnetic effect on bioconvection of a nanofluid along a vertical stretching sheet. They concluded that the ratio of convective to conductive heat transfer across (normal to) the boundary decreases with the magnetic parameter increasing, while the density number of the motile microorganisms decreases with magnetic parameter enlarging. Their work indicates that the MHD flow could be used to alter the distributions of microorganisms in potential applications such as removal of microorganisms attached in a water tank.

In this paper, we shall consider an unsteady bioconvection squeezing flow in a horizontal channel in the presence of both chemical reaction and magnetic effect. In a flowing fluid that contains heat transfer, mass transfer, and chemical reactions, the flow velocity is always to affect partially the transport of reactants, products, and heat and hence influences the overall mass and heat transfer rates. An analysis for accurate estimates of the transport effects upon the performance of processes with combined chemical reaction and magnetic effect in a combined flow regime is thus very desirable. As far as we know, no such study has been available in the literature. This study attempts to meet such a need.

\section{Mathematical Descriptions}

Consider the unsteady MHD flow of an electrically conducting fluid containing motile microorganisms between two infinite parallel plates in the presence of a first-order chemical reaction. As shown in Figure 1, the coordinate system is chosen such that the $x$-axis is along the lower plate and $y$ axis is perpendicular to the main flow direction, respectively. It is assumed that the two plates are placed at the distance $y=h(t)=[\nu(1-a t) / b]^{1 / 2}$ while the upper plate is moving towards or away from the lower stationary plate with the

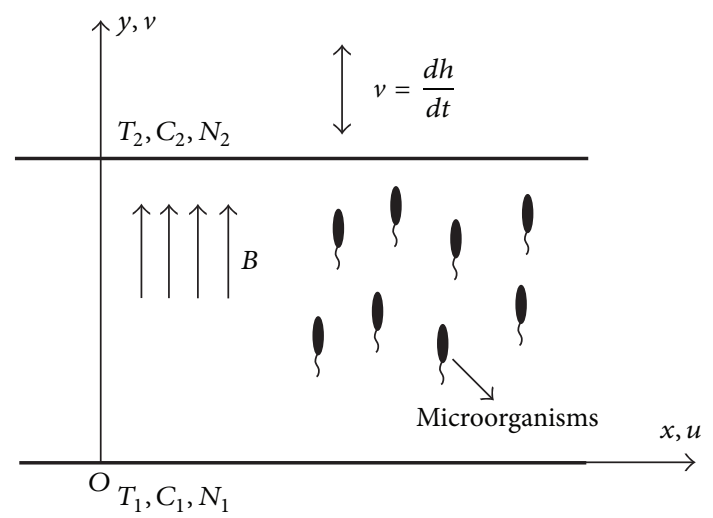

FIgure 1: Physical model and coordinate system.

velocity $v(t)=d h / d t$. Here, $a$ and $b$ are positive numbers, $v$ is the kinetic viscosity, and $t$ denotes the time. Obviously, it is known that $1-a t$ must be greater than zero, which indicates that $a$ must be less than $1 / t$. Physically, when $a=0$, it means that the two plates are fixed. As $0<a<1 / t$, it shows that the upper plate is squeezed to the lower one. While as $a<0$, it indicates the upper plate moves away from the lower plate. It is also assumed that the lower and upper plates are maintained, respectively, at the constant temperatures $T_{1}$ and $T_{2}$, constant concentration of microorganisms $N_{1}$ and $N_{2}$, and constant chemical reaction concentrations $C_{1}$ and $C_{2}$. The time-varying magnetic field of strength $B(t)$ is imposed along the $y$-axis. Based on the above assumptions, the conservation equations for total mass, momentum, thermal energy, mass diffusion, and microorganisms equations are expressed by

$$
\begin{aligned}
\nabla \cdot \mathbf{V} & =0 \\
\frac{\partial \mathbf{V}}{\partial t}+(\mathbf{V} \cdot \nabla) \mathbf{V} & =-\frac{1}{\rho} \nabla p+\nu \nabla^{2} \mathbf{V}+\frac{1}{\rho}(\mathbf{J} \times \mathbf{B}), \\
\frac{\partial T}{\partial t}+\mathbf{V} \cdot \nabla T & =\alpha \nabla^{2} T+\frac{Q_{s}(t)}{\rho c_{p}}\left(T-T_{0}\right), \\
\frac{\partial C}{\partial t}+\mathbf{V} \cdot \nabla C & =D \nabla^{2} C-K(t)\left(C-C_{0}\right), \\
\frac{\partial N}{\partial t} & =-\nabla \cdot \mathbf{j},
\end{aligned}
$$

where $\mathbf{V}$ is the velocity vector, $\mathbf{J}$ is the electric current density in the fluid, $\mathbf{B}$ is the magnetic induction, $\rho$ is the fluid density, $p$ is the pressure, $v$ is the kinematic viscosity of the fluid, $T$ is the temperature, $T_{0}$ is the reference temperature, $\alpha$ is the thermal diffusivity, $Q_{s}(t)$ is the volumetric rate of heat generation/absorption, $C$ is the concentration of the chemical reaction, $C_{0}$ is the reference concentration, $D$ is the mass diffusivity, $K(t)$ is the reaction rate, $N$ is the concentration of motile microorganism, and $\mathbf{j}$ is the flux of microorganisms.

It is assumed that the magnetic Reynolds number is small («1) so that the induced magnetic field can be omitted. It is also assumed that the heating due to the plates and the chemical reaction is weak so that microorganisms can survive 
and their gyrotactic behaviour can keep unchanged. With those assumptions, we can expand (1) into the following forms:

$$
\begin{aligned}
& \frac{\partial \xi}{\partial t}+u \frac{\partial \xi}{\partial x}+v \frac{\partial \xi}{\partial y}=v\left(\frac{\partial^{2} \xi}{\partial x^{2}}+\frac{\partial^{2} \xi}{\partial y^{2}}\right)+\frac{\sigma B^{2}(t) u}{\rho}, \\
& \frac{\partial T}{\partial t}+u \frac{\partial T}{\partial x}+v \frac{\partial T}{\partial y} \\
& =\alpha\left(\frac{\partial^{2} T}{\partial x^{2}}+\frac{\partial^{2} T}{\partial y^{2}}\right)+\frac{Q_{s}(t)}{\rho c_{p}}\left(T-T_{0}\right), \\
& \frac{\partial C}{\partial t}+u \frac{\partial C}{\partial x}+v \frac{\partial C}{\partial y} \\
& =D\left(\frac{\partial^{2} C}{\partial x^{2}}+\frac{\partial^{2} C}{\partial y^{2}}\right)-K(t)\left(C-C_{0}\right), \\
& \frac{\partial N}{\partial t}+u \frac{\partial N}{\partial x}+v \frac{\partial N}{\partial y}+\frac{\partial}{\partial x}(N \widetilde{v})+\frac{\partial}{\partial y}(N \widetilde{v}) \\
& =D_{n}\left(\frac{\partial^{2} N}{\partial x^{2}}+\frac{\partial^{2} N}{\partial y^{2}}\right)
\end{aligned}
$$

subject to the boundary conditions

$$
\begin{gathered}
u=0, \\
v=0, \\
T=T_{1}, \\
C=C_{1}, \\
N=N_{1} \\
u=0, \\
v=\frac{d h}{d t}, \\
T=T_{2}, \\
C=C_{2}, \\
N=N_{2}
\end{gathered}
$$$$
\text { at } y=0 \text {, }
$$

$$
\text { at } y=h(t) \text {, }
$$

where $u$ and $v$ are the velocity components in the $x$ - and $y$ directions defined by $u=\partial \psi / \partial y$ and $v=-\partial \psi / \partial x$ with $\psi$ being the stream function, $\xi=-\nabla^{2} \psi$ is the vorticity function, $N$ is the density of motile microorganisms, $\widetilde{v}=\left[b_{c} W_{c} /\left(C_{1}-\right.\right.$ $\left.\left.C_{0}\right)\right] \partial C / \partial y$ is the average swimming velocity vector of the oxytactic microorganisms with $b_{c}$ being the chemotaxis constant and $W_{c}$ being the maximum cell swimming speed, and $D_{n}$ is the diffusivity of microorganisms.
We then introduce the following similarity transformations and nondimensional quantities:

$$
\begin{aligned}
\psi(x, y) & =\left(\frac{b v}{1-a t}\right)^{1 / 2} x f(\eta), \\
u & =\frac{b x}{1-a t} f^{\prime}(\eta), \\
v & =-\left(\frac{b v}{1-a t}\right)^{1 / 2} f(\eta), \\
\eta & =\left(\frac{b}{v(1-a t)}\right)^{1 / 2} y, \\
\theta(\eta) & =\frac{T-T_{0}}{T_{1}-T_{0}}, \\
\phi(\eta) & =\frac{C-C_{0}}{C_{1}-C_{0}} \\
w(\eta) & =\frac{N}{N_{1}} .
\end{aligned}
$$

In view of (4), (2) can be reduced to the following nondimensional form:

$$
\begin{aligned}
& f^{\prime \prime \prime \prime}(\eta)-M f^{\prime}(\eta)-3 \beta f^{\prime \prime}(\eta)-\beta \eta f^{\prime \prime \prime}(\eta) \\
& -f^{\prime}(\eta) f^{\prime \prime}(\eta)+f(\eta) f^{\prime \prime \prime}(\eta)=0, \\
& \frac{1}{\operatorname{Pr}} \theta^{\prime \prime}(\eta)+Q \theta(\eta)-\beta \eta \theta^{\prime}(\eta)+f(\eta) \theta^{\prime}(\eta)=0, \\
& \frac{1}{\operatorname{LePr}} \phi^{\prime \prime}(\eta)-K_{0} \phi(\eta)-\beta \eta \phi^{\prime}(\eta)+f(\eta) \phi^{\prime}(\eta)=0, \\
& w^{\prime \prime}(\eta)-\operatorname{Sc} \beta \eta w^{\prime}(\eta)+\operatorname{Sc} f(\eta) w^{\prime}(\eta) \\
& -\operatorname{Pe} w(\eta) \phi^{\prime \prime}(\eta)-\operatorname{Pe} \phi^{\prime}(\eta) w^{\prime}(\eta)=0 .
\end{aligned}
$$

subject to the following boundary conditions:

$$
\begin{gathered}
f^{\prime}(0)=0, \\
f(0)=0, \\
\theta(0)=1, \\
\phi(0)=1, \\
w(0)=1, \\
f^{\prime}(1)=0, \\
f(1)=\beta, \\
\theta(1)=\delta_{\theta}, \\
\phi(1)=\delta_{\phi}, \\
w(1)=\delta_{w},
\end{gathered}
$$

where $M$ is the magnetic field parameter, $\beta$ is the squeezing parameter, Pr is the Prandtl number, $Q$ is the heat generation/absorption parameter, Le is the Lewis number, $K_{0}$ is the 
chemical reaction parameter, $\mathrm{Sc}$ is the Schmidt number, $\mathrm{Pe}$ is the bioconvection Péclet number, and $\delta_{\theta}, \delta_{\phi}$, and $\delta_{w}$ are constants, which are defined by

$$
\begin{aligned}
M & =\frac{\sigma}{\nu \rho} B_{0}^{2}, \\
\beta & =\frac{a}{2 b}, \\
\operatorname{Pr} & =\frac{\nu}{\alpha}, \\
Q & =\frac{Q_{0}}{\rho c_{p}}, \\
\mathrm{Le} & =\frac{\alpha}{D}, \\
K_{0} & =\left(\frac{b}{1-a t}\right)^{-1} K(t), \\
\mathrm{Sc} & =\frac{\nu}{D_{n}}, \\
\mathrm{Pe} & =\frac{b_{c} W_{c}}{D_{n}}, \\
\delta_{\theta} & =\frac{T_{2}-T_{0}}{T_{1}-T_{0}}, \\
\delta_{\phi} & =\frac{C_{2}-C_{0}}{C_{1}-C_{0}}, \\
\delta_{w} & =\frac{N_{2}}{N_{1}} .
\end{aligned}
$$

In above equation $B_{0}=(b / \nu(1-a t))^{-3 / 4} B(t)$ is a reference magnetic field parameter, $Q_{0}=(b /(1-a t))^{-1} Q_{s}(t)$ is a reference heat generation/absorption parameter, and $K_{0}$ is the chemical reaction parameter.

Practical interests of this problem are the local skin friction $C_{f x}$, the local Nusselt number $\mathrm{Nu}_{x}$, the local wall mass flux $\mathrm{Sh}_{x}$, and the local wall motile microorganisms flux $Q_{x}$, which are defined by

$$
\begin{aligned}
C_{f x} & =\frac{\tau_{w}}{(1 / 2) \rho U_{x}^{2}}, \\
\mathrm{Nu}_{x} & =\frac{x q_{w}}{k\left(T_{1}-T_{0}\right)}, \\
\mathrm{Sh}_{x} & =\frac{x j_{w}}{D\left(C_{1}-C_{0}\right)}, \\
Q_{x} & =\frac{x h_{w}}{D_{n}\left(N_{1}-N_{0}\right)},
\end{aligned}
$$

where

$$
\begin{gathered}
\tau_{w}=\mu\left(\frac{\partial u}{\partial y}\right)_{y=0}, \\
q_{w}=-k\left(\frac{\partial T}{\partial y}\right)_{y=0},
\end{gathered}
$$

$$
\begin{aligned}
& j_{w}=-D\left(\frac{\partial C}{\partial y}\right)_{y=0}, \\
& h_{w}=-D_{n}\left(\frac{\partial N}{\partial y}\right)_{y=0} .
\end{aligned}
$$

Without loss of generality, we set $N_{0}=0$. Substituting (12) into (11), we obtain

$$
\begin{aligned}
\operatorname{Re}_{x}^{1 / 2} C_{f x} & =2 f^{\prime \prime}(0), \\
\mathrm{Re}_{x}^{-1 / 2} \mathrm{Nu}_{x} & =-\theta^{\prime}(0), \\
\operatorname{Re}_{x}^{-1 / 2} \mathrm{Sh}_{x} & =-\phi^{\prime}(0), \\
\operatorname{Re}_{x}^{-1 / 2} \mathrm{Q}_{x} & =-w^{\prime}(0),
\end{aligned}
$$

where $\operatorname{Re}_{x}=U_{w} x / v$ is the local Reynolds number.

\section{Results and Discussion}

Equations (5)-(8) and their boundary conditions (9) are solved numerically for various values of the parameters using the shooting method along with fourth-order Runge-Kutta scheme. In the computation, the value of the step size $\Delta \eta$ between 0.001 and 0.01 was used, in order that the numerical values obtained are mesh independent. In addition, we choose $\delta_{\theta}=1 / 2, \delta_{\phi}=0$, and $\delta_{w}=1$ for all considered cases, which are not against physical requirements. Further, to check the accuracy of the results, we compare them with those given by the BVPh2.0 programme (which is based on the homotopy analysis methods technique). Here, the initial approximations $f_{0}(\eta), \theta_{0}(\eta), \phi_{0}(\eta)$, and $w_{0}(\eta)$ are chosen based on (9) as below:

$$
\begin{aligned}
& f_{0}(\eta)=3 \beta \eta^{2}-2 \beta \eta^{3}, \\
& \theta_{0}(\eta)=1+\left(\delta_{\theta}-1\right) \eta, \\
& \phi_{0}(\eta)=1+\left(\delta_{\phi}-1\right) \eta, \\
& w_{0}(\eta)=1+\left(\delta_{w}-1\right) \eta .
\end{aligned}
$$

The auxiliary linear operator are chosen as:

$$
\begin{array}{r}
L[f(\eta)]=\frac{\partial^{4} f(\eta)}{\partial \eta^{4}}, \\
L[\theta(\eta)]=\frac{\partial^{2} \theta(\eta)}{\partial \eta^{2}}, \\
L[\phi(\eta)]=\frac{\partial^{2} \phi(\eta)}{\partial \eta^{2}}, \\
L[w]=\frac{\partial^{2} w(\eta)}{\partial \eta^{2}} .
\end{array}
$$

Through calculation and comparison, excellent agreement is found, as shown in Figure 2. 


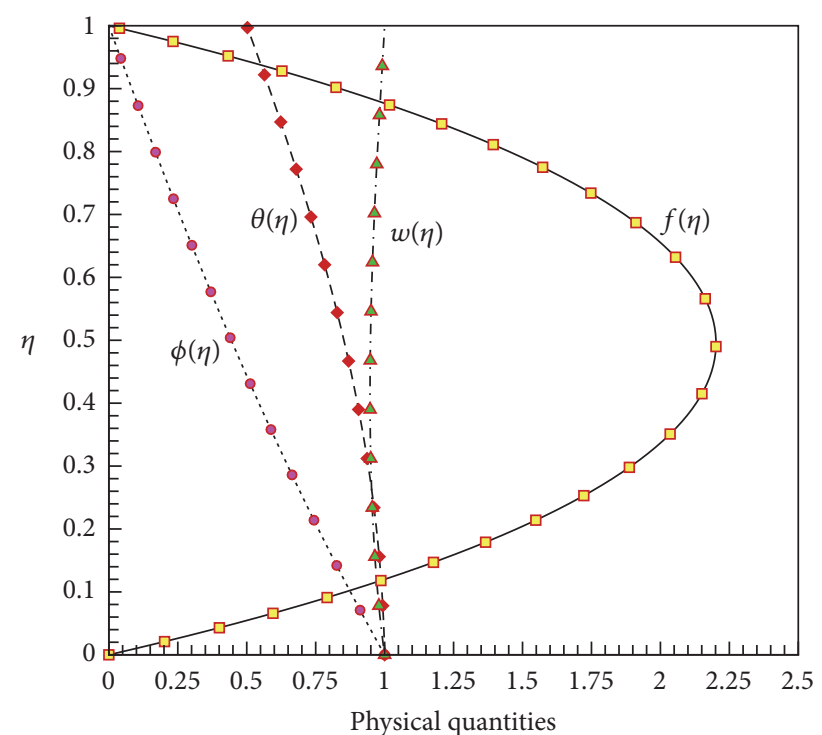

FIgURE 2: Comparison of the BVPh2.0 programme results and the numerical results for $\beta=1.5, Q=1, K_{0}=1$, and $\operatorname{Pr}=\mathrm{Le}=\mathrm{Sc}=$ $\mathrm{Pe}=1$, where symbols are numerical results and lines are $\mathrm{BVPh} 2.0$ programme results.

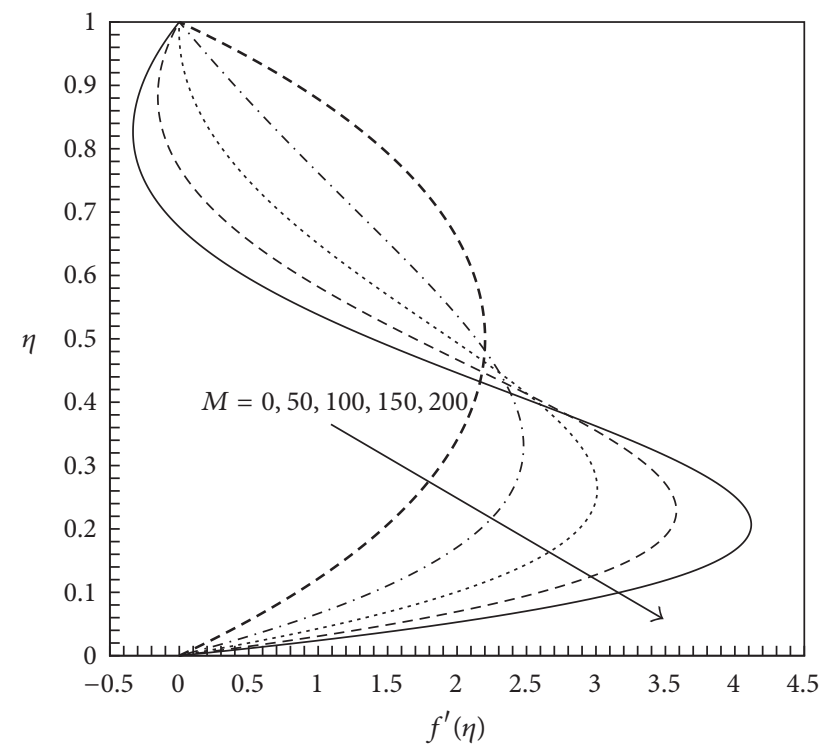

FIgURE 3: Variation of $f^{\prime}(\eta)$ with $\eta$ for various $M$ in the case of $\beta=$ $1.5, \mathrm{Q}=1, K_{0}=1$, and $\mathrm{Pr}=\mathrm{Le}=\mathrm{Sc}=\mathrm{Pe}=1$.

We first consider the influence of the magnetic parameter $M$ on variations of various profiles. It is shown in Figure 3 that $f^{\prime}(\eta)$ develops rapidly with $M$ evolving, the enlargement of $M$ causes the enhancement of the flow velocity in the vicinity of the lower plate but the reduction of the flow velocity on the other side. This is due to the fact that the enhanced Lorentz force imparts additional momentum into the squeezing channel, which accelerates the flow motion in the lower part of the channel. Synchronously the flow near the upper plate is retarded owing to the nonslip boundary. It is found in Figure 4 that the temperature profiles decrease

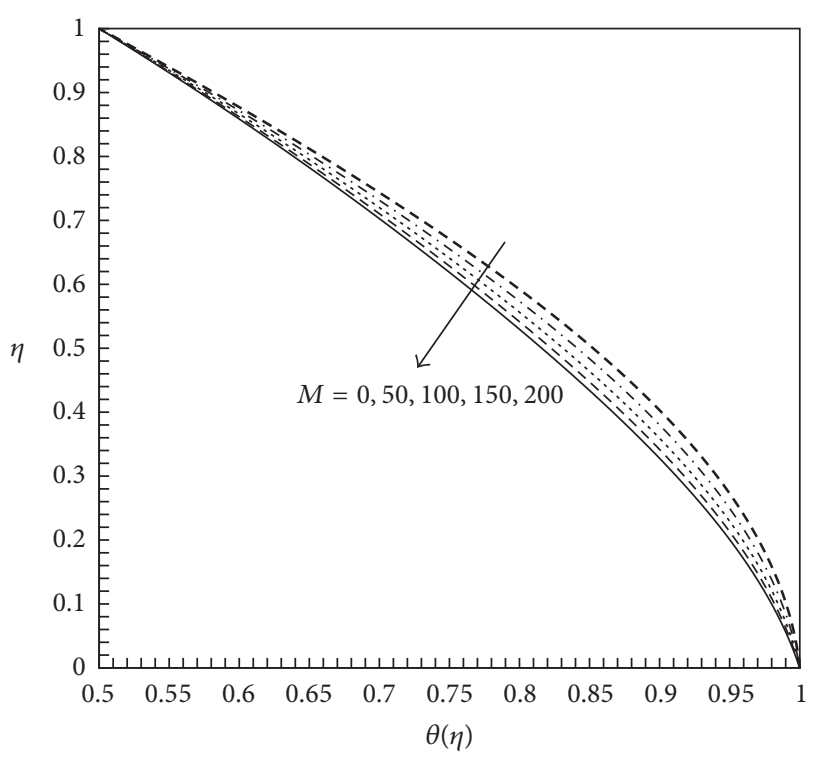

FIgURE 4: Variation of $\theta(\eta)$ with $\eta$ for various $M$ in the case of $\beta=$ 1.5, $Q=1, K_{0}=1$, and $\operatorname{Pr}=\mathrm{Le}=\mathrm{Sc}=\mathrm{Pe}=1$.

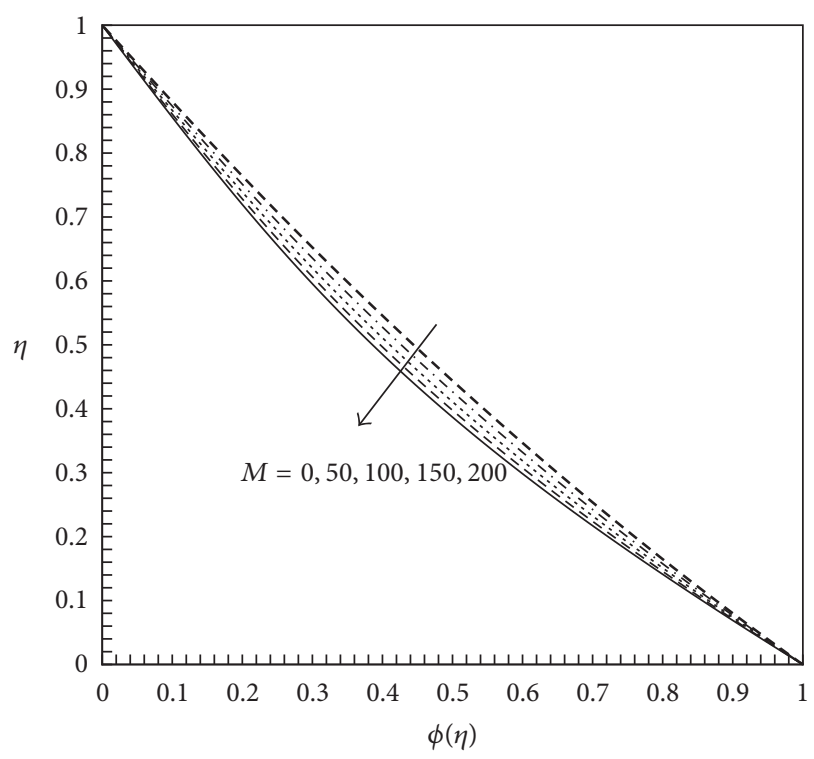

FIGURE 5: Variation of $\phi(\eta)$ with $\eta$ for various $M$ in the case of $\beta=$ 1.5, $\mathrm{Q}=1, K_{0}=1$, and $\mathrm{Pr}=\mathrm{Le}=\mathrm{Sc}=\mathrm{Pe}=1$.

continuously as $M$ increases. The reason is that the enhanced Lorentz force increases the thermal boundary-layer thickness in the channel. Particularly, we notice that the temperature profiles near the lower plate reduce a bit more quickly than that near the upper plate, which is due to the fact that the flow velocity in the vicinity of the lower plate is higher than that near the upper plate. As shown in Figure 5, the influence of the magnetic parameter $M$ on the concentration of chemical reaction $\phi(\eta)$ is similar to that on the temperature profiles $\theta(\eta)$; that is to say, the larger the value of $M$, the lower the profile $\phi(\eta)$. Physically, this variation is mainly caused by the mass transportation induced by the enhanced Lorentz 


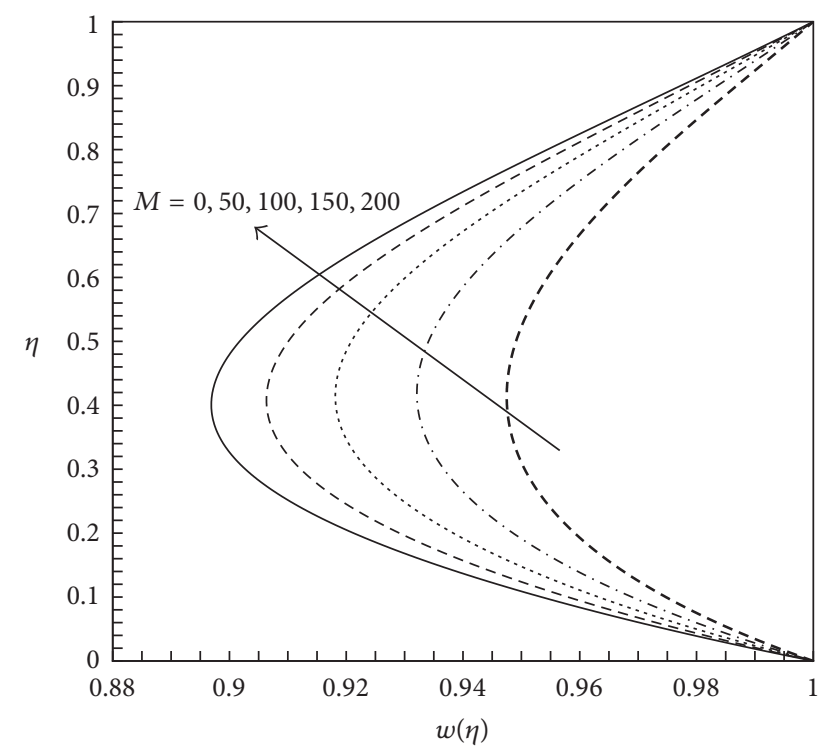

FIGURE 6: Variation of $w(\eta)$ with $\eta$ for various values of parameter $M$ in the case of $\beta=1.5, Q=1, K_{0}=1$, and $\mathrm{Pr}=\mathrm{Le}=\mathrm{Sc}=\mathrm{Pe}=1$.

force. It is illustrated in Figure 6 that $M$ has obvious effect on variation of the motile density of the microorganisms $w(\eta) . w(\eta)$ decreases as $M$ enlarges, since the increase of $M$ accelerates the fluid motion, which is helpful to alter the distributions of microorganisms.

We next consider the effects of the chemical reaction parameter $K_{0}$ and the heat generation/absorption parameter $Q$ on various profiles. In view of (5), (6), and (7), it is readily found that $f(\eta)$ and $\theta(\eta)$ are uncoupled with $\phi(\eta)$; that is to say, the variation of $\phi(\eta)$ due to the evolvement of $K_{0}$ does not affect the velocity and temperature distributions at all. Therefore, we need only to consider the influence of $K_{0}$ on $\phi(\eta)$ and $w(\eta)$. As shown in Figure 7, the increase of $K_{0}$ causes the decrease of $\phi(\eta)$. Physically, $K_{0}$ is related to the chemical reaction rate. The larger this value is, the faster the reaction rate is. As a result, the concentration of the chemical species is reduced as $K_{0}$ evolves. It is illustrated in Figure 8 that the density of motile microorganisms $w(\eta)$ reduces as $K_{0}$ enlarges. This is due to the fact that the enhancement of $K_{0}$ reduces the concentration of chemical species, which leads to the decrease of the density of motile microorganisms. Similarly, it is found from (5)-(8) that the heat generation/absorption parameter $Q$ is only associated with $\theta(\eta)$ via the reduced energy equation $(8)$ and $\theta(\eta)$ has no relation with any other functions. We therefore only need to consider its influence on the temperature distribution. As shown in Figures 9 and 10, for $Q>0$, corresponding to the heat source generation case, $\theta(\eta)$ increase rapidly with $Q$ increasing. On the other hand, for $Q<0$, corresponding to the heat source absorption case, $\theta(\eta)$ reduces rapidly with $Q$ decreasing. In other words, the heat generation/absorption parameter $Q$ has a great influence on the temperature.

We now discuss the effects of various physical parameters on physically important quantities. It is known that $M$ and $\delta_{\theta}$ can affect the local Nusselt number $\mathrm{Re}_{x}^{-1 / 2} \mathrm{Nu}_{x}$, according to

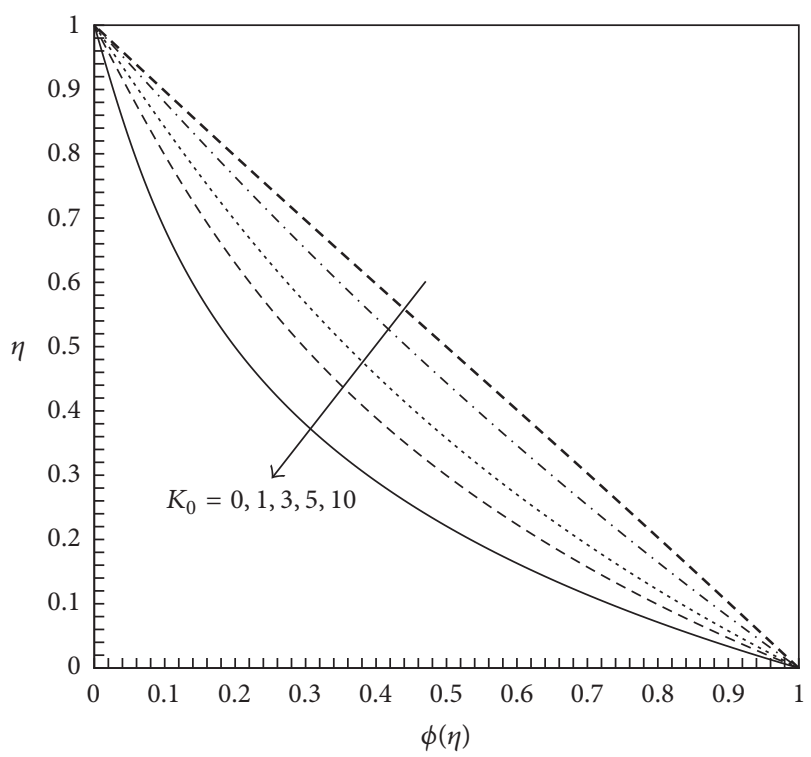

FIgURE 7: Variation of $\phi(\eta)$ with $\eta$ for various values of $K_{0}$ in the case of $\beta=1.5, Q=1, M=1$, and $\operatorname{Pr}=\mathrm{Le}=\mathrm{Sc}=\mathrm{Pe}=1$.

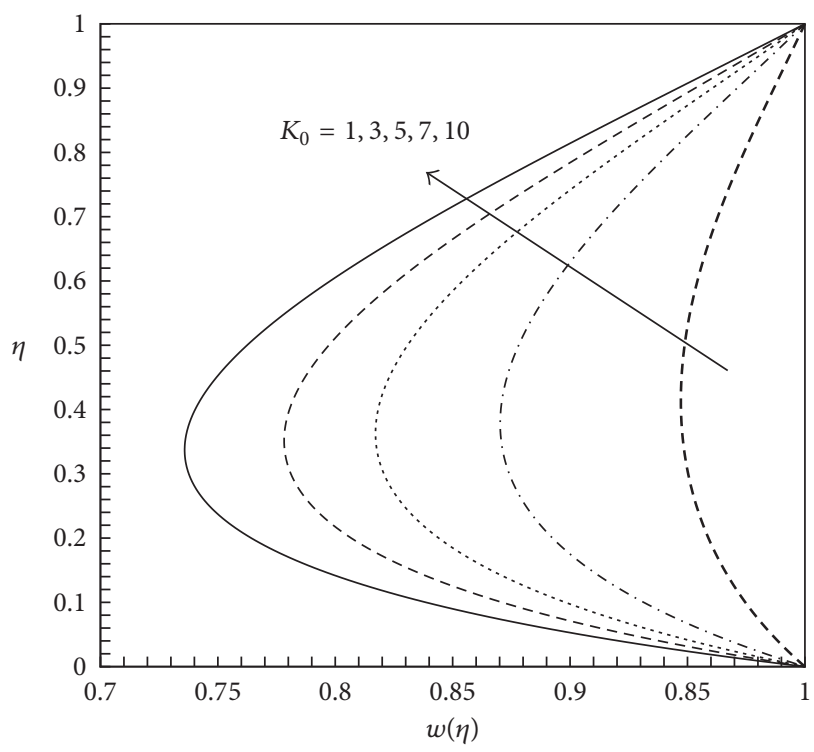

Figure 8: Variation of $w(\eta)$ with $\eta$ for various values of $K_{0}$ in the case of $\beta=1.5, Q=1, M=1$, and $\mathrm{Pr}=\mathrm{Le}=\mathrm{Sc}=\mathrm{Pe}=1$.

(6) and its boundary condition (9) for temperature distribution on the upper plate. As shown in Table 1, the local Nusselt number increases gradually as the magnetic parameter $M$ enlarges. This is due to the fact that the increase of $M$ means more additional momentum caused by the enhanced Lorentz force is imparted into the sneezing channel. As a result, the fluid flow is accelerated and the temperature difference between this plate and the reference value is increased. Similarly, we know that $M$ and $\delta_{\phi}$ take effects on the local wall mass flux $\mathrm{Re}_{x}^{-1 / 2} \mathrm{Sh}_{x}$. It is seen in Table 2 that the local wall mass flux increases continuously as $M$ enlarges. This trend indicates that the magnetic parameter plays a role on 


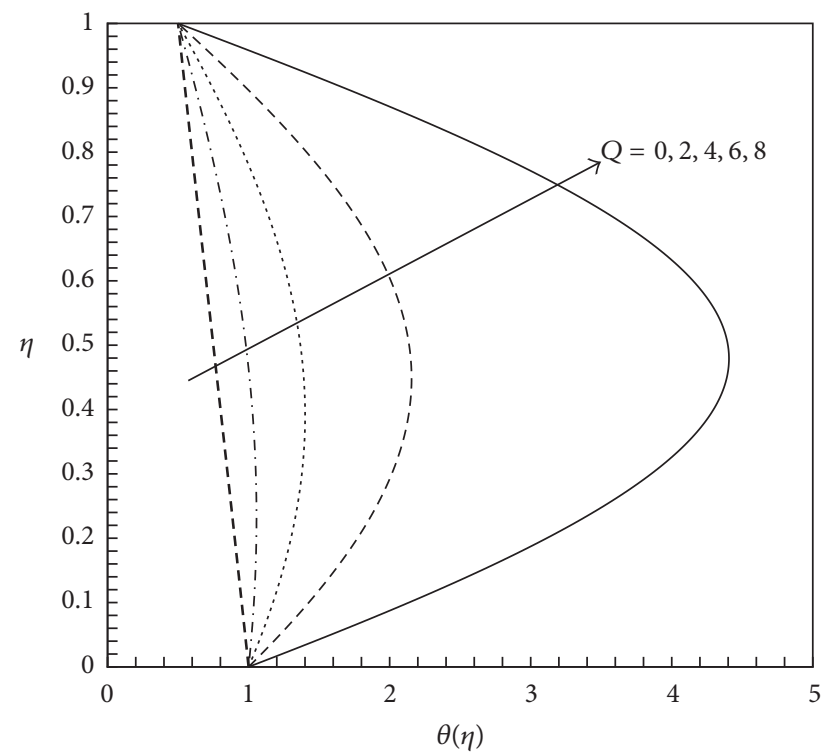

FIGURE 9: Variation of $\theta(\eta)$ with $\eta$ for various positive values of $Q$ in the case of $\beta=1.5, M=1, K_{0}=1$, and $\mathrm{Pr}=\mathrm{Le}=\mathrm{Sc}=\mathrm{Pe}=1$.

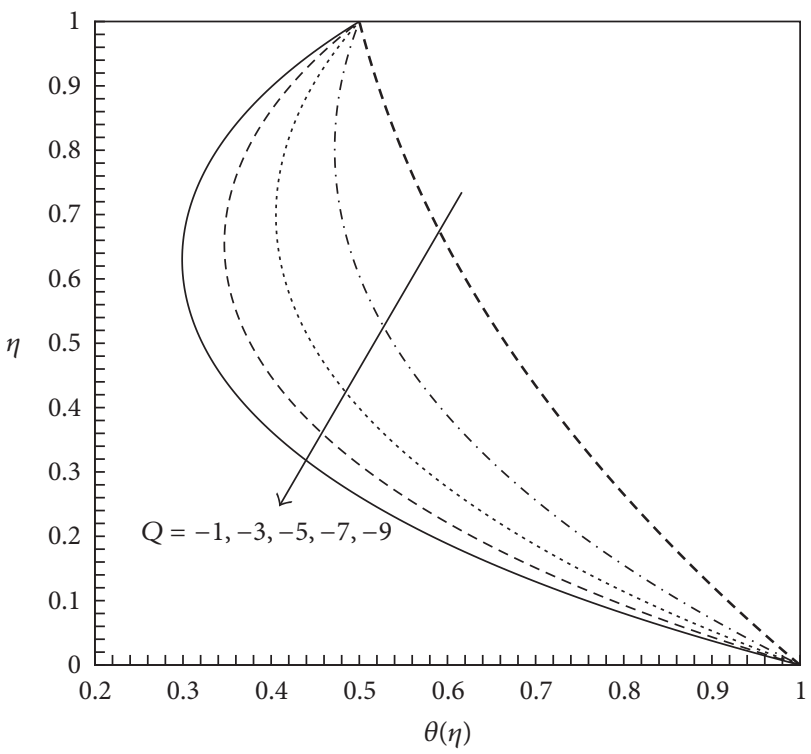

FIGURE 10: Variation of $\theta(\eta)$ with $\eta$ for various negative values of $Q$ in the case of $\beta=1.5, M=1, K_{0}=1$, and $\operatorname{Pr}=\mathrm{Le}=\mathrm{Sc}=\mathrm{Pe}=1$.

variation of the nanoparticles distribution. When $M$ is fixed, it is found in this table that the local wall mass flux reduces gradually as $\delta_{\phi}$ evolves.

In practical application, It is interesting to give some approximate formulae for calculating those physical quantities. For instance, by regression analysis, we obtain the estimated formulae for various physical quantities as functions of $M$ and/or $K_{0}$ in the case of $Q=\operatorname{Pr}=\mathrm{Le}=\mathrm{Sc}=\mathrm{Pe}=1$ and $\beta=1.5$,

$$
\begin{aligned}
\operatorname{Re}_{x}^{1 / 2} C_{f x} & =9.035322+0.177944 M, \\
\operatorname{Re}_{x}^{-1 / 2} \mathrm{Nu}_{x} & =0.04904+0.00524 M,
\end{aligned}
$$

TABLE 1: The local Nusselt number $\mathrm{Re}_{x}^{-1 / 2} \mathrm{Nu}_{x}$ for various values of parameters $\delta_{\theta}$ and $M$ in the case of $\delta_{\phi}=0, \delta_{w}=1, Q=K_{0}=\operatorname{Pr}=$ $\mathrm{Le}=\mathrm{Sc}=\mathrm{Pe}=1$, and $\beta=1.5$.

\begin{tabular}{lcccc}
\hline$\delta_{\theta}$ & $M=0$ & $M=50$ & $M=100$ & $M=200$ \\
\hline 0 & 0.62504 & 0.68927 & 0.75182 & 0.85656 \\
0.2 & 0.39363 & 0.44398 & 0.49285 & 0.57423 \\
0.4 & 0.16221 & 0.19868 & 0.23388 & 0.29191 \\
0.6 & -0.06921 & -0.04661 & -0.02509 & 0.00959 \\
0.8 & -0.30062 & -0.29191 & -0.28406 & -0.27273 \\
1 & -0.53204 & -0.53721 & -0.54303 & -0.55505 \\
\hline
\end{tabular}

TABLE 2: For different $M$, the values of the local wall flux $\operatorname{Re}_{x}^{-1 / 2} \mathrm{Sh}_{x}$ for various values of parameter $\delta_{\phi}$ in the case of $\delta_{\theta}=0.5, \delta_{w}=1$, $Q=K_{0}=\operatorname{Pr}=\mathrm{Le}=\mathrm{Sc}=\mathrm{Pe}=1$, and $\beta=1.5$.

\begin{tabular}{lcccc}
\hline$\delta_{\phi}$ & $M=0$ & $M=50$ & $M=100$ & $M=200$ \\
\hline 0 & 1.28437 & 1.33808 & 1.39126 & 1.48271 \\
0.2 & 1.11792 & 1.16167 & 1.20508 & 1.28000 \\
0.4 & 0.95147 & 0.98525 & 1.01890 & 1.07729 \\
0.6 & 0.78501 & 0.80883 & 0.83271 & 0.87459 \\
0.8 & 0.61856 & 0.63242 & 0.64653 & 0.67188 \\
1 & 0.45210 & 0.45600 & 0.46035 & 0.46918 \\
\hline
\end{tabular}

$$
\begin{aligned}
\operatorname{Re}_{x}^{-1 / 2} \mathrm{Sh}_{x}= & 1.1097864+0.0081446 M \\
& +0.20924432 K_{0}, \\
\operatorname{Re}_{x}^{-1 / 2} Q_{x}= & 0.1403712+0.00089064 M \\
& +0.22188504 K_{0} .
\end{aligned}
$$

Similarly, we are able to give the estimated formulae by linear regression for other physical parameters. For instance, we are able to obtain the approximate expressions regarding $\delta_{\theta}, \delta_{\phi}$ and $\delta_{w}$ by defining

$$
P_{h \mathrm{Q}}=B_{0}+B_{1} \delta_{\theta}+B_{2} \delta_{\phi}+B_{3} \delta_{w}
$$

where $P_{h \mathrm{Q}}$ can be replaced by various quantities including the local Nusselt number, the local wall mass flux, and the local wall motile microorganisms. When the value of $\delta_{\theta}, \delta_{\phi}$, and $\delta_{w}$ are, respectively, taken as $0,0.4,0.8,1.2,1.6$, an 2 , the coefficients $B_{1}, B_{2}$, and $B_{3}$ can be computed by linear regression, as shown in Tables 3-5. Note that here we set $Q=\operatorname{Pr}=\mathrm{Le}=\mathrm{Sc}=\mathrm{Pe}=K_{0}=1$ and $\beta=1.5$.

\section{Conclusions}

The unsteady squeezing flow of an incompressible viscous fluid with suspension of small motile microorganisms between two infinite parallel plates in the presence of both chemical reaction and magnetic effect has been studied. The governing equations embody the total mass, momentum, thermal energy, and mass diffusion and microorganisms are reduced to a set of nonlinear ordinary differential equations via a set of similarity transformations. The effects of the 
TABLE 3: Linear regression coefficients and the maximum relative error $\varepsilon$ for the local Nusselt number $\mathrm{Re}_{x}^{-1 / 2} \mathrm{Nu}_{x}$.

\begin{tabular}{lccccc}
\hline$M$ & $B_{0}(y$-intercept $)$ & $B_{1}$ & $B_{2}$ & $B_{3}$ & $\varepsilon$ \\
\hline 0 & 0.6250 & -1.1571 & 0 & 0 & $5.64 E-07$ \\
50 & 0.6893 & -1.2265 & 0 & 0 & $5 E-07$ \\
100 & 0.7518 & -1.2949 & 0 & 0 & $1.57 E-16$ \\
200 & 0.8566 & -1.4116 & 0 & 0 & $5 E-07$ \\
500 & 0.9942 & -1.5765 & 0 & 0 & $5 E-07$ \\
\hline
\end{tabular}

TABLE 4: Linear regression coefficients and the maximum relative error $\varepsilon$ for the local wall flux $\operatorname{Re}_{x}^{-1 / 2} \mathrm{Sh}_{x}$.

\begin{tabular}{lccccc}
\hline$M$ & $B_{0}(y$-intercept $)$ & $B_{1}$ & $B_{2}$ & $B_{3}$ & $\varepsilon$ \\
\hline 0 & 1.2844 & 0 & -0.8323 & 0 & $5.39 E-07$ \\
50 & 1.3381 & 0 & -0.8821 & 0 & $4.59 E-07$ \\
100 & 1.3913 & 0 & -0.9309 & 0 & $5.82 E-07$ \\
200 & 1.4827 & 0 & -1.0135 & 0 & $5.39 E-07$ \\
500 & 1.6144 & 0 & -1.1258 & 0 & $5.12 E-07$ \\
\hline
\end{tabular}

TABLE 5: Linear regression coefficients and the maximum relative error $\varepsilon$ for the local wall motile microorganisms flux $\operatorname{Re}_{x}^{-1 / 2} Q_{x}$.

\begin{tabular}{lccccc}
\hline$M$ & $B_{0}(y$-intercept $)$ & $B_{1}$ & $B_{2}$ & $B_{3}$ & $\varepsilon$ \\
\hline 0 & 1.3019 & 0 & 0.1535 & -1.0013 & 0.0401 \\
50 & 1.4114 & 0 & 0.1082 & -1.0534 & 0.0407 \\
100 & 1.5201 & 0 & 0.0628 & -1.1044 & 0.0411 \\
200 & 1.7075 & 0 & -0.0158 & -1.1907 & 0.0419 \\
500 & 1.9784 & 0 & -0.1263 & -1.3083 & 0.0431 \\
\hline
\end{tabular}

various parameters on the distributions of velocity, temperature, chemical reaction concentration, and density of motile microorganisms, as well as the important physical quantities, have been examined in detail. The major contributions can be summarized as follows:

(i) This problem solved by two methods and the results obtained by numerical methods have good agreement with the analytical solution.

(ii) The magnetic parameter $M$ takes actions on each profile since it imparts additional momentum into the squeezing channel. The increase of $M$ leads to the enhancement of the velocity profile, but causes the decrease of the profiles of the temperature, the chemical reaction concentration, and the motile density of microorganisms. In short, the magnetic field can be used to control the flow characteristics.

(iii) The chemical parameter $K_{0}$ plays a role in the chemical reaction concentration and motile density of microorganisms profiles. The increase of $K_{0}$ causes the decrease of both profiles.

(iv) The heat generation/absorption parameter $Q$ is critical for the profiles of the temperature. The temperature is found to increase as we go from strong absorption to strong generation of heat. (v) The estimated expressions for physical quantities of practical interests are given by linear regression for a range of parameters.

\section{Competing Interests}

The authors declare that no potential competing financial interests with others exist.

\section{Authors' Contributions}

Hang Xu and Qingkai Zhao conceived the work that led to the submission. Qingkai Zhao prepared figures. Hang Xu played an important role in interpreting the results. Longbin Tao drafted and revised the manuscript. All authors reviewed the manuscript and approved the final version.

\section{Acknowledgments}

The second author would like to acknowledge the program for New Century Excellent Talents in University (Grant no. NCET-12-0347) for its financial support.

\section{References}

[1] J. Stefan, "Versucheüber die scheinbare adhäsion," Akadenie deer Wissenschaften in Wien, Math.-Naturw, vol. 69, pp. 713721, 1874.

[2] D. F. Moore, "A review of squeeze films," Wear, vol. 8, no. 4, pp. 245-263, 1965.

[3] P. S. Gupta and A. S. Gupta, "Squeezing flow between parallel plates," Wear, vol. 45, no. 2, pp. 177-185, 1977.

[4] H. M. Duwairi, B. Tashtoush, and R. A. Damseh, "On heat transfer effects of a viscous fluid squeezed and extruded between two parallel plates," Heat and Mass Transfer, vol. 41, no. 2, pp. 112-117, 2004.

[5] R. L. Verma, "A numerical solution for squeezing flow between parallel channels," Wear, vol. 72, no. 1, pp. 89-95, 1981.

[6] M. Mustafa, T. Hayat, and S. Obaidat, "On heat and mass transfer in the unsteady squeezing flow between parallel plates," Meccanica, vol. 47, no. 7, pp. 1581-1589, 2012.

[7] T. Hayat, A. Qayyum, F. Alsaadi, M. Awais, and A. M. Dobaie, "Thermal radiation effects in squeezing flow of a Jeffery fluid," European Physical Journal Plus, vol. 128, no. 8, pp. 85-91, 2013.

[8] T. J. Pedley, N. A. Hill, and J. O. Kessler, "The growth of bioconvection patterns in a uniform suspension of gyrotactic micro-organisms," Journal of Fluid Mechanics, vol. 195, pp. 223237,1988

[9] T. J. Pedley and J. O. Kessler, "Hydrodynamic phenomena in suspensions of swimming microorganisms," Annual Review of Fluid Mechanics, vol. 24, pp. 313-358, 1992.

[10] A. V. Kuznetsov, "The onset of bioconvection in a suspension of gyrotactic microorganisms in a fluid layer of finite depth heated from below," International Communications in Heat and Mass Transfer, vol. 32, no. 5, pp. 574-582, 2005.

[11] A. V. Kuznetsov, "The onset of nanofluid bioconvection in a suspension containing both nanoparticles and gyrotactic microorganisms," International Communications in Heat and Mass Transfer, vol. 37, no. 10, pp. 1421-1425, 2010. 
[12] H. Xu and I. Pop, "Mixed convection flow of a nanofluid over a stretching surface with uniform free stream in the presence of both nanoparticles and gyrotactic microorganisms," International Journal of Heat and Mass Transfer, vol. 75, pp. 610623, 2014

[13] A. V. Kuznetsov and D. A. Nield, "The Cheng-Minkowycz problem for natural convective boundary layer flow in a porous medium saturated by a nanofluid: a revised model," International Journal of Heat and Mass Transfer, vol. 65, pp. 682685, 2013.

[14] H. Xu and I. Pop, "Fully developed mixed convection flow in a horizontal channel filled by a nanofluid containing both nanoparticles and gyrotactic microorganisms," European Journal of Mechanics. B. Fluids, vol. 46, pp. 37-45, 2014.

[15] A. Raees, H. Xu, and S.-J. Liao, "Unsteady mixed nanobioconvection flow in a horizontal channel with its upper plate expanding or contracting," International Journal of Heat and Mass Transfer, vol. 86, pp. 174-182, 2015.

[16] H. Alfvén, "Existence of electromagnetic-hydrodynamic waves," Nature, vol. 150, no. 3805, pp. 405-406, 1942.

[17] A. Chakrabarti and A. S. Gupta, "Hydromagnetic flow and heat transfer over a stretching sheet," Q Appl Math, vol. 37, no. 1, pp. 73-78, 1979.

[18] K. Vajravelu and A. Hadjinicolaou, "Convective heat transfer in an electrically conducting fluid at a stretching surface with uniform free stream," International Journal of Engineering Science, vol. 35, no. 12-13, pp. 1237-1244, 1997.

[19] H. A. Attia and N. A. Kotb, "MHD flow between two parallel plates with heat transfer," Acta Mechanica, vol. 117, pp. 215-220, 1996.

[20] K. A. Helmy, "HMD unsteady free convection flow past AA vertical porous plate," Zeitschrift für Angewandte Mathematik und Mechanik, vol. 78, no. 4, pp. 255-270, 1998.

[21] W. I. Thacker, L. T. Watson, and S. Kishore Kumar, "Magnetohydrodynamic free convection from a disk rotating in a vertical plane," Applied Mathematical Modelling, vol. 14, no. 10, pp. 527$535,1990$.

[22] H. A. Attia, "Unsteady MHD flow and heat transfer of dusty fluid between parallel plates with variable physical properties," Applied Mathematical Modelling, vol. 26, no. 9, pp. 863-875, 2002.

[23] H. S. Takhar, A. J. Chamkha, and G. Nath, "Flow and mass transfer on a stretching sheet with a magnetic field and chemically reactive species," International Journal of Engineering Science, vol. 38, no. 12, pp. 1303-1314, 2000.

[24] Z. G. Makukula, P. Sibanda, S. S. Motsa, and S. Shateyi, "On new numerical techniques for the MHD flow past a shrinking sheet with heat and mass transfer in the presence of a chemical reaction," Mathematical Problems in Engineering, vol. 2011, Article ID 489217, 19 pages, 2011.

[25] A. Zeeshan, R. Ellahi, and M. Hassan, "Magnetohydrodynamic flow of water/ethylene glycol based nanofluids with natural convection through a porous medium," European Physical Journal Plus, vol. 129, no. 261, 2014.

[26] M. Bhatti, A. Zeeshan, and R. Ellahi, "Simultaneous effects of coagulation and variable magnetic field on peristaltically induced motion of Jeffrey nanofluid containing gyrotactic microorganism," Microvascular Research, vol. 110, pp. 32-42, 2017.

[27] R. Ellahi, M. Hassan, and A. Zeeshan, "Study of natural convection mhd nanofluid by means of single and multi-walled carbon nanotubes suspended in a salt-water solution," IEEE Transactions on Nanotechnology, vol. 14, no. 4, pp. 726-734, 2015.

[28] W. A. Khan and O. D. Makinde, "MHD nanofluid bioconvection due to gyrotactic microorganisms over a convectively heat stretching sheet," International Journal of Thermal Sciences, vol. 81, no. 1, pp. 118-124, 2014. 


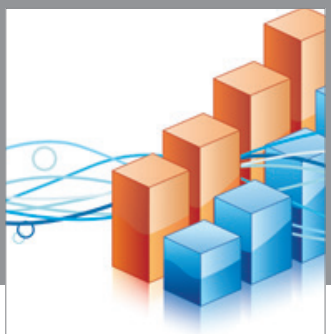

Advances in

Operations Research

vatem alat4

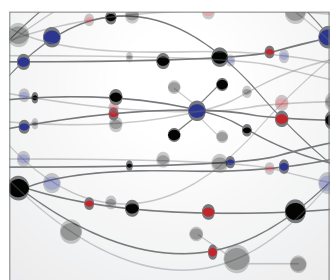

\section{The Scientific} World Journal
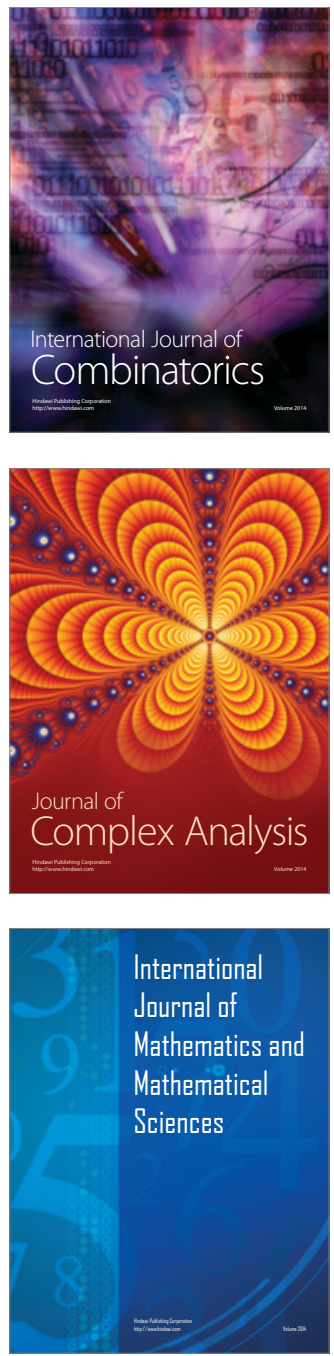
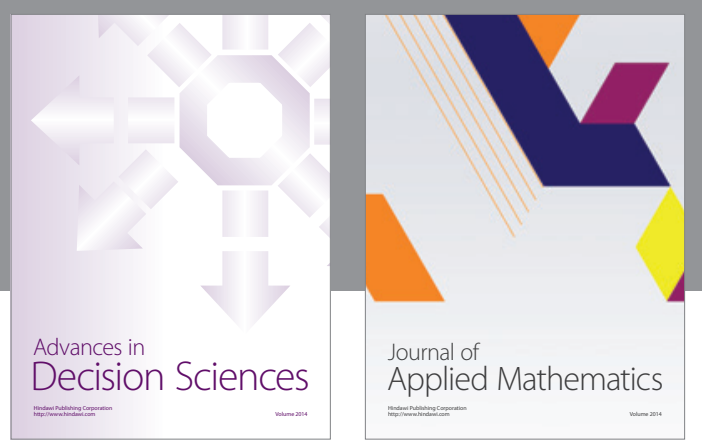

Algebra

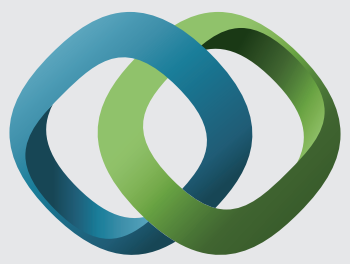

\section{Hindawi}

Submit your manuscripts at

https://www.hindawi.com
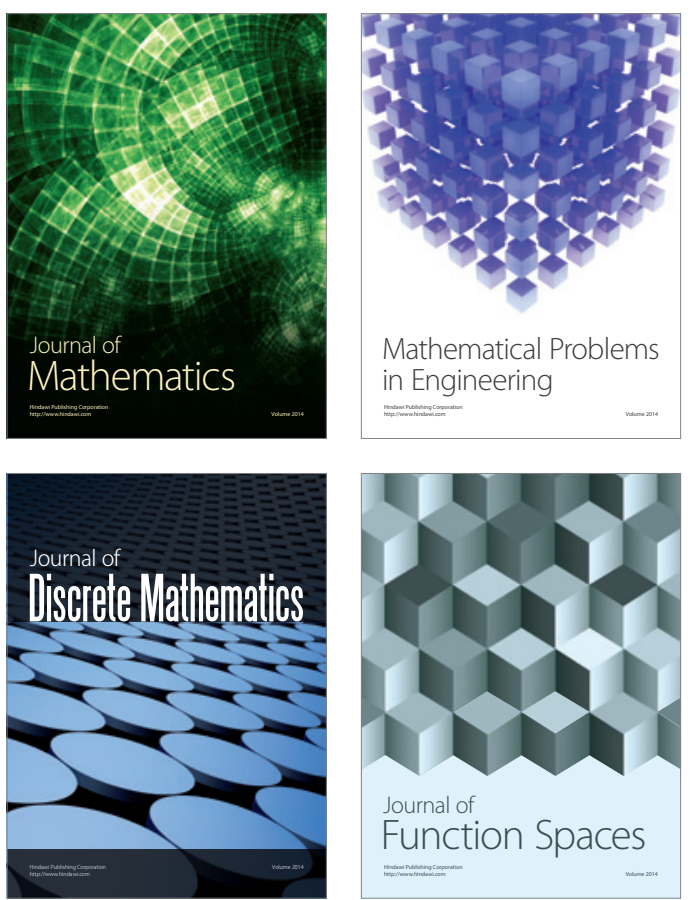

Mathematical Problems in Engineering
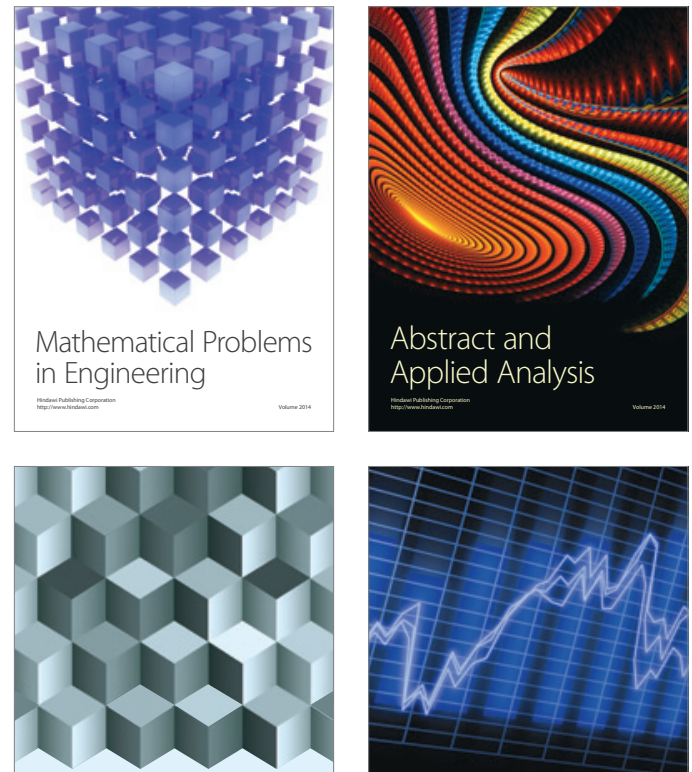

Journal of

Function Spaces

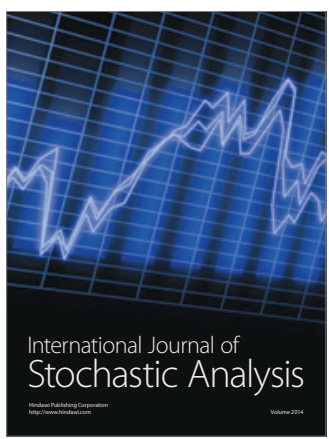

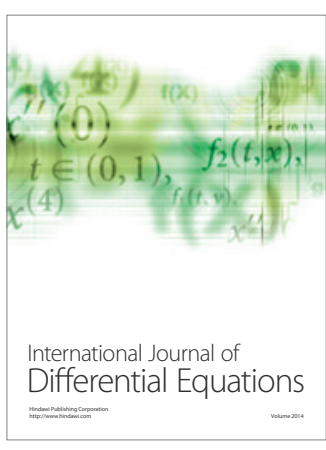
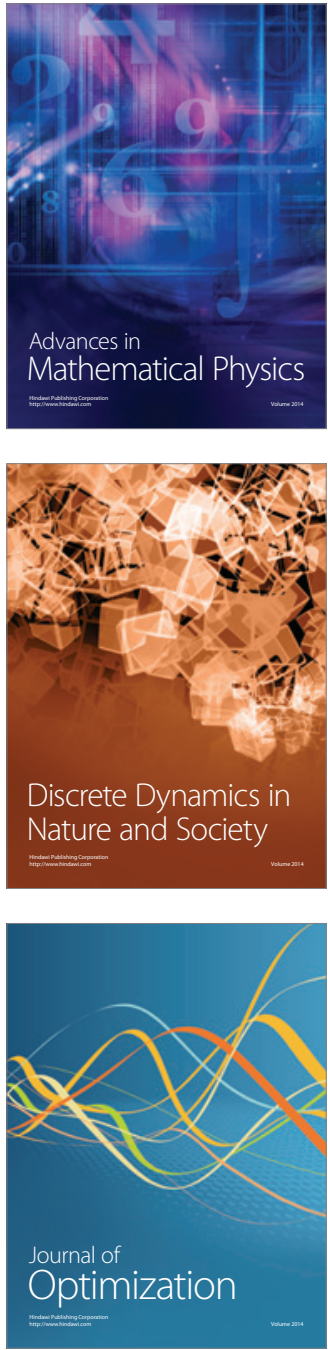